Fuertes-Olivera, P. A. y Bergenholtz, H. (2018). «Diccionarios del español para la producción de textos», en RILEX. Revista sobre investigaciones léxicas, 1/I, pp. 5-28.

\title{
DICCIONARIOS DEL ESPAÑOL PARA LA PRODUCCIÓN DE TEXTOS
}

\author{
Pedro A. Fuertes-Olivera \\ Centro Internacional de Lexicografía (Universidad de Valladolid) \\ Department of Afrikaans and Dutch, University of Stellenbosh \\ pedro@emp.uva.es \\ Henning Bergenholtz \\ Centre for Lexicography (Universidad de Aarbus) \\ Department of Afrikaans and Dutch, University of Stellenbosh \\ Department of Information Science, University of Pretoria \\ hbe@.cc.au.dk
}

\section{RESUMEN}

$\mathrm{El}$ diccionario del español para la producción de textos es una herramienta de consulta diseñada para ayudar a un usuario tipo, por ejemplo un ser humano o un programa informático, a solucionar problemas de redacción. Este diccionario tiene en abril de 2017 alrededor de 80.000 acepciones descritas (aumenta el número de las mismas a una media de 30.000 acepciones por año) y es un integrante de un portal lexicográfico con objetivos comerciales, Diccionarios Valladolid-UVa. El diccionario presenta novedades teóricas y prácticas basadas en la idea de que un diccionario de Internet debe ser un diccionario dinámico, es decir, un diccionario que ofrece varias posibilidades de consulta gracias a la interrelación de los tres componentes básicos de un diccionario de Internet: (a) una base de datos lexicográfica o editor; (b) uno o varios motores de búsqueda; (c) una o varias interfaces. Como los paquetes informáticos comerciales existentes en la actualidad no permiten el desarrollo de este tipo de diccionarios, nuestros diccionarios se diseñan y construyen con tecnologías propias pensadas para favorecer el proceso de construcción, actualizar los datos lexicográficos al minuto, reusar los datos lexicográficos existentes y hacer uso tanto de tecnologías propias (por ejemplo, sistemas de búsquedas maximizadoras) como de libre disposición (por ejemplo Ngram Viewer).

Palabras clave: producción de textos, diccionario de Internet dinámico, español, Teoría Funcional de la Lexicografía, lexicografía comercial

\begin{abstract}
Dictionaries for text production are information tools that are designed and constructed for helping users (both humans and machines) to encode both oral and written texts. The diccionario del español para la producción de textos is one of these information tools. It will be part of Diccionarios Valladolid-UVa, a commercially-driven dictionary portal which aims to fill a gap in the Spanish lexicographical landscape in which dictionaries such as these are difficult to find. Our dictionary is innovative and puts into practice some new concepts and ideas, especially those associated with the interrelationship of the three basic components of online dictionaries (a) the editor; (b) the search engine(s); and (c) the interface(s). As commercially available dictionary writing systems do not offer flexibility for designing and making dynamic dictionaries, our dictionaries use both free (Ngram Viewer) and own technologies with the aim of making them informatively sound, economically viable, reusable, and easy to update, for example, this dictionary can be updated in minutes.
\end{abstract}

Keywords: text production, Function Theory of Lexicography, online dynamic dictionary, Spanish, commercial lexicography 


\section{INTTRODUCCIÓN}

La llegada de Internet ha tenido varias repercusiones importantes en el campo de la lexicografía (Fuertes-Olivera, 2016, 2018; Fuertes-Olivera \& Bergenholtz, 2011a; Granger \& Paquot, 2012; Jackson, 2013); al menos tres de ellas son importantes para este artículo. La primera está relacionada con la naturaleza de la lexicografía, que podemos definir como la teoría y práctica de los diccionarios, es decir de las herramientas de consulta puntual que se diseñan y construyen para satisfacer las necesidades de información que un usuario potencial pueda tener en una o varias situaciones extralexicográficas en las que acude al diccionario para solucionar sus necesidades de información de la forma más fácil y rápida posible.

La segunda repercusión es que la llegada de Internet a la lexicografía también debe verse como un ejemplo más de innovación distuptiva, es decir, la introducción de tecnologías que llevan consigo la desaparición de productos y servicios tal y como son conocidos en un momento determinado y su sustitución por otros, que son diferentes aunque pudieran mantener el mismo nombre. Además del producto y servicio también sufre modificación el modelo de negocio que sustenta tal producto o servicio (Christensen, 1997). En el caso del diccionario como producto reconocido de la lexicografía es un hecho que la mayoría de las editoriales que publicaban diccionarios impresos o bien han dejado de hacerlo, o bien solo sacan al mercado adaptaciones o actualizaciones de sus diccionarios anteriores. Esto ha ocurrido básicamente en la primera etapa de innovación disruptiva que va dirigida a los usuarios menos exigentes, por ejemplo a los usuarios que acceden a los diccionarios de acceso libre, como los que encuentran en portales como WordReference.com. En estos sitios web estos usuarios tienen a su alcance varias posibilidades de consulta que ofrecen al usuario datos procedentes normalmente de diccionarios impresos, como los que obtenemos al consultar cachupin, ina en WordReference.com (ejemplo 1): simplemente se define el lema con un sinónimo, algo que también hace para este lema el Diccionario de la lengua española (2014: s.v. gachupin):

cachupin, ina

1. m. y f. gachupin

Ejemplo 1. cachupin, ina en WordReference.com 
Los teóricos de la innovación disruptiva (Christensen, 1997) también nos dicen que existe una segunda fase, que sigue a la fase de bajas prestaciones. Esta segunda fase se denomina fase de tecnologías de nuevo mercado. En esta segunda fase, los innovadores van mejorando y adaptando sus productos y servicios ofreciendo mejoras claras sin poner en riesgo la naturaleza del producto o servicio, por ejemplo, respetando la sencillez, facilidad, accesibilidad y conveniencia que debe tener cualquier herramienta de consulta. El resultado de esta segunda fase de la innovación disruptiva es la aparición de productos y servicios que transforman por completo un sector generando otro nuevo que, aunque pueda seguir manteniendo el nombre tradicional, es completamente diferente. Esto está ocurriendo en la actualidad en lexicografía, siendo esta la tercera repercusión de la llegada de Internet a la lexicografía.

Esta tercera repercusión consiste en el diseño y construcción de diccionarios nuevos. Hay dos líneas de trabajo encaminadas a conseguir diccionarios diferentes a los existentes hasta la fecha. Una de las líneas no ha logrado hasta el momento ofrecer productos comerciales. Sigue en la fase de investigación y está centrada en descubrir algoritmos con los que poder llevar a cabo tareas lexicográficas de forma automática o semiautomática; un buen ejemplo es el proyecto DBpedia, que es una comunidad colaborativa centrada en extraer datos estructurados de Wikipedia con el fin de lograr que los mismos se conviertan en información, es decir, sean útiles para usuarios humanos o máquinas. En este artículo no vamos a seguir analizando esta línea de trabajo; hasta la fecha tanto sus planteamientos teóricos como sus resultados prácticos están en fase experimental.

La otra línea de investigación apuesta por desarrollar diccionarios diferentes, como los integrantes del portal lexicográfico Diccionarios Valladolid-UVa que se espera que vean la luz en el año 2018 o 2019. Uno de los diccionarios integrantes de este portal es el Diccionario del español para la producción de textos que analizamos en este artículo. Para poder construir diccionarios diferentes, es decir, diccionarios que pudieran ocupar el nicho de mercado dejado por los diccionarios tradicionales, es necesario saber qué es lo que existe en el mercado lexicográfico en la actualidad y decidir si lo que existe se puede mejorar o modificar con el fin de crear un producto o servicio lexicográfico con 
potencial comercial. Es decir, el análisis de los diccionarios existentes (secciones 2 y 3 de este artículo) debe desembocar en una idea o concepto de diccionario de Internet viable e informativamente rentable, como veremos en las secciones 4 y 5 al referirnos a los diccionarios del portal Diccionarios Valladolid-UV a. Dichos diccionarios se han diseñado teniendo en cuenta el estado actual de la investigación lexicográfica, especialmente la lexicografía de Internet, y con un objetivo comercial, es decir con la intención de obtener recursos económicos que, primero cubran los costes de producción (alrededor de 5 euros por cada significado descrito) y, segundo, ofrezcan una rentabilidad en línea con las actividades económicas propias de la economía basada en el conocimiento (alrededor de un 20\% anual del capital invertido).

\section{DICCIONARIOS PARA LA PRODUCCIÓN DE TEX'TOS}

Varios investigadores (por ejemplo, Marello, 2003) han señalado que los diccionarios de producción son bastante frecuentes, especialmente aquellos diseñados para ayudar a los aprendices de una lengua extranjera a redactar textos en esa lengua. Quizás el diccionario de producción más conocido sea el Longman Language Activator (Summers, 1993), comercializado como “The World's First Production Dictionary" (el primer diccionario de producción del mundo). En su Introducción se afirma que

El Longman Language Activator es un diccionario de ideas y de cómo las mismas pueden expresarse en inglés. Es un nuevo tipo de diccionario cuyo objetivo es ayudar al estudiante de nivel intermedio o de nivel avanzado a producir lengua, es decir a codificar sus ideas (Longman Language Activator 1993, Página F8; traducción propia).

En realidad, el Longman Language Activator es un diccionario con una concepción o planta lexicográfica combinada; es un diccionario con una función comunicativa primaria (ayudar a escribir textos en inglés) y con una función cognitiva secundaria (ayudar a mejorar el conocimiento de la lengua inglesa por parte del estudiante). El subtítulo "El Primer Diccionario de Producción de Textos del Mundo" es solo un eslogan publicitario ya que al menos existen diccionarios con estas dos funciones desde hace unos 300 años. Por ejemplo, Hornby (1963) ya escribía en la introducción del Advanced Learner's Dictionary of Current English que el diccionario tenía como objetivo "ayudar a componer en inglés ("help in the compilation of English"). En resumen, 
diccionarios como el Longman Language Activator señalan que hay una tradición lexicográfica que construye diccionarios para la producción. Estos diccionarios lematizan conceptos que van seguidos de definiciones en forma de glosa para cada uno de los significados del concepto; a continuación se incluyen palabras de la lengua que ilustran el significado del concepto seguidas de una breve descripción lexicográfica que normalmente incluye pronunciación, información gramatical de la palabra y una breve explicación de la misma con ejemplos de uso. Por ejemplo, al concepto inglés carry, el Longman Language Activator le reserva cuatro significados, uno de los cuales aparece descrito con la glosa "designed to be carried" (diseñado para ser transportado), seguido por portable, que aparece descrito lexicográficamente a continuación (ejemplo 2):

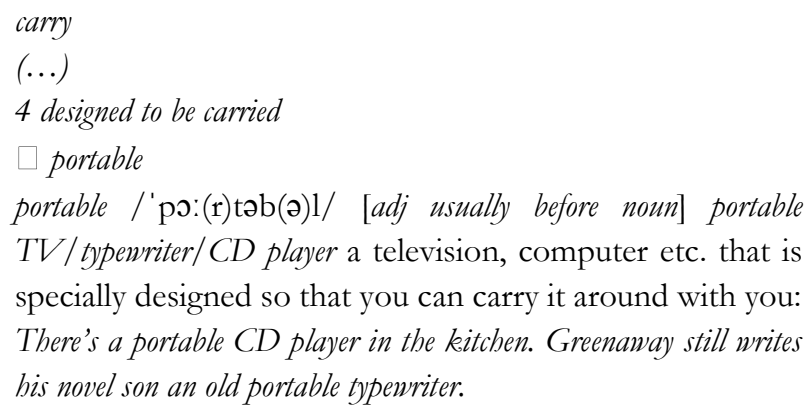

Ejemplo 2. Descripción de uno de los significados de carry en el Longman Language Activator

\section{DICCIONARIOS DEL ESPAÑOL PARA LA PRODUCCIÓN DE T'EX'TOS}

Una revisión crítica de algunas publicaciones recientes sobre lexicografía española por ejemplo, Bosque y Rodríguez Barrios (2018), Ahumada, (2014), Haensch y Omeñaca (2004) y Kiesler (2013) — parece apuntarnos que en la tradición lexicográfica española diccionarios como el Longman Language Activator o bien son una rareza o bien no tienen un objetivo tan claro y preciso. Bosque y Barrios Rodríguez (2018) se refieren a diccionarios parecidos a los que la tradición lexicográfica española suele identificar como diccionario ideológico, tesauro, o atlas léxico. La principal característica de estos diccionarios es que van del concepto al significante o palabra y por ello exigen del usuario una gran competencia lexicográfica, terminológica y lingüística. Por ejemplo, el diccionario ideológico de la lengua española (Casares, 1942) comprende varios textos iniciales - entre ellos un texto para justificar el concepto lexicográfico empleado y el significado de las abreviaturas empleadas — y tres partes principales: (a) parte sinóptica: 
en esta parte se incluye la clasificación ideológica del diccionario, es decir ofrece un esquema de los grupos, categorías y subcategorías bajo los cuales se ha organizado el vocabulario descrito en el diccionario; (b) parte analógica: en esta parte los conceptos se distribuyen en grupos de palabra en los que hay sinónimos, antónimos, conceptos afines y otras palabras de su familia léxica. El traslado de los conceptos a enunciados se hace siguiendo un orden claro y bien establecido. Por ejemplo, los sustantivos de un concepto van seguidos por sinónimos, aumentativos, diminutivos, posesivos, etc.; (c) parte alfabética: se explica el significado de cada palabra y su pertenencia a uno de los grupos de la parte analógica.

Además de estos diccionarios para la producción (también son diccionarios para la producción los diccionarios de sinónimos, antónimos, de pronunciación, ortografía, gramática, etc., que no trataremos en este artículo por razones de espacio) conviene analizar con más detalle una serie de diccionarios polifuncionales españoles que tienen gran prestigio en la lexicografía española y han tenido una gran difusión: Gran diccionario de uso del español actual (Sánchez Pérez, 2001); Diccionario de uso del español (María Moliner, 2007); Diccionario del español actual (Seco y otros, 2011); Diccionarios.com, zona Premium). Todos estos son diccionarios polifuncionales y nuestro análisis se centrará principalmente en su posible utilidad para la producción de textos en español.

\subsection{GRAN DICCIONARIO DE USO DEL ESPAÑOL ACTUAL}

El Gran diccionario de uso del español actual es un diccionario general y pedagógico, es decir, es un diccionario pensado para satisfacer al mismo tiempo las necesidades del hablante español nativo y del estudiante de este idioma, tal y como veremos a continuación. En la Introducción (pp. 7-14) se describen las características del diccionario, siendo relevantes para este artículo su compromiso con describir "la lengua usada en nuestros días" (p. 7). La utilización del adjetivo "usada" indica al lector que este diccionario también tiene interés en describir no solo el significado de un lema sino también su uso. Es por ello un diccionario que incluye datos lexicográficos para ayudar a componer textos orales o escritos. Entre estos datos destacan los siguientes: información gramatical básica (por ejemplo, la clase de palabra); ejemplos de uso (todos ellos proceden del corpus Cumbre); información gramatical avanzada, como la 
referida al régimen preposicional de verbos, sustantivos y adjetivo, y a las formas irregulares de los verbos; marcas diafásicas, diatópicas y diastráticas; locuciones y expresiones; y sinónimos y antónimos.

Además, y esto debe resaltarse por su importancia para la producción y como característica específica del diccionario, el diccionario ofrece un tratamiento preciso de la ortografía del español, especialmente de la separación silábica de los lemas y de fin de línea. Este tratamiento responde a criterios objetivables descritos de manera precisa:

1. la combinación consonante + vocal debe mantenerse integra, por ejemplo cons $\bullet \tan \bullet$ te;

2. los "compuestos en los que la etimología aparece como clara o evidente, se separan donde termina uno de sus componentes: des $\bullet$ ha $\bullet e r$. Si la etimología es percibida por los hablantes como menos evidente, se admite tanto la separación por etimología como por conjuntos silábicos $(\mathrm{C}+\mathrm{V})$ : no $\bullet 0 \bullet$ tros $/$ nos $\bullet \bullet^{\bullet}$ tros ..." (p. 11);

3. nunca se dejará una vocal única al final de la palabra, aunque constituya sílaba, como Ma•ría;

4. los grupos vocálicos átonos "no se separarán nunca, en cualquier posición en

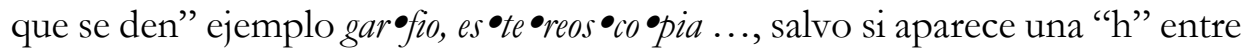
las vocales que constituye el grupo (en $\bullet$ de he $\bullet$ sar) o si se trata de un “compuesto de clara etimología” (en •tre • • bier •to) (p. 11);

5. en los grupos vocálicos formados por tónica + átona o átona + tónica nunca se separan si las vocales 'i, u' "forman parte de dicho grupo" (p. 11) (por ejemplo, cie $\bullet$ ); se separan si las vocales "que intervienen en el grupo son la 'a, e, o', en cualquiera de sus posibles combinaciones y la segunda vocal va seguida de una consonante" (p. 11), como por ejemplo lo $\bullet$ ar,

6. los triptongos nunca se separan: des $\bullet$ pre $\bullet$ ciáis $\bullet$

7. se separan los prefijos que aparecen en secuencias de consonante-vocalconsonante + vocal-consonante: des $\bullet$ em $\bullet$ pol $\bullet$ var;

8. las grafías “ch, rr, ll” nunca deben separarse (be $\bullet b o)$. 


\subsection{DICCIONARIO DE USO DEL ESPAÑOL}

En el Prólogo a la tercera edición del Diccionario de uso del español, Manuel Seco resalta que este diccionario es un instrumento para la codificación:

La otra meta fundamental-muy raramente intentada por otros, debido a su dificultad: solo tenía como gran precedente el Diccionario ideológico de Julio Casares - era la consulta "bidireccional". El de Moliner, con método propio, ofrecía una doble utilidad: por una parte, era un instrumento, como los diccionarios usuales, para comprender o descifrar lo que se oye o lee (un diccionario descodificador); pero al mismo tiempo era también un instrumento para cifrar o componer lo que se quiere expresar (un diccionario codificador). Un objetivo sumamente valioso, aunque menos valorado por los lectores que los antes enumerados. (cursivas en el original; Seco, 2007: XI-XII).

La comparación de la reflexión anterior de Seco con los datos incluidos en el listado del diccionario, así como con las explicaciones ofrecidas en los textos iniciales y finales —en concreto, los textos "La Tercera edición del Diccionario de María Moliner" (pp. XIIIXVII), y "Uso del Diccionario" (pp. XXI-XXXVI) - nos permite concluir que el diccionario es un diccionario polifuncional que incluye el mayor número de datos posibles, sin discriminar entre las diferentes situaciones de uso. Es decir, el diccionario obliga al usuario a decidir por sí mismo cuál o cuáles de los datos tratados lexicográficamente puede necesitar en la situación de producción. Es por ello que en el diccionario encontramos entradas con datos totalmente necesarios en una situación de producción (por ejemplo, la explicación de la conjugación del verbo desviar en el ejemplo 3) con otras entradas — como cantarera y cantarero, - a (ejemplo 4) — que tienen datos para la producción pero exigen un gran esfuerzo cognitivo al usuario del diccionario, ya que debe decidir por su cuenta el valor de los mismos (por ejemplo, por qué cantarera de cantarero, - $a$ es diferente de cantarera):

\section{desviar}

$\square$ CONJUG. La $i$ de la raíz es tónica en los presentes de indicativo y subjuntivo y en el imperativo, salvo en la $2 .^{a}$ persona del singular correspondiente a la forma vos, en la $1 .{ }^{a}$ persona del plural y en la 2. ${ }^{a}$ persona del plural correspondiente a la forma vosotros: 'desvío, desvías, desvía, desvían; desvíe, desvíes, desvíe, desvíen; desvía, desvíe, desvíen'. En el resto de las formas la $i$ es átona, y se pronuncia generalmente sin formar diptongo con la vocal que le sigue. $\Rightarrow$ Apend. II, PRONUNCIACIÓN (verbos en -iar).

Ejemplo 3. Notas de uso y Conjugación en el Diccionario de uso del español

cantarera $\mathrm{f}$. Pollo de albañilería o armazón de madera que se ponen los cántaros. $\Rightarrow$ Zafariche cantarero, - a (de cántaro) n. Alfarero.

Ejemplo 4. Entrada con datos para la producción confusos en el Diccionario de uso del español 
La reflexión de Seco quizás cobre mayor sentido al observar que el diccionario incluye datos similares a los que reflejamos para el Gran diccionario de uso del español actual así como varios apéndices con datos pensados para la producción; por ejemplo, el apéndice II se asemeja a un manual de gramática (tiene 100 páginas) y está centrado en analizar y explicar reglas y excepciones de aspectos centrales de la gramática del español, como son los siguientes: (a) la acentuación; (b) el adjetivo (por ejemplo, todos los adjetivos españoles pueden nominalizarse de una forma fácil y sencilla); (c) el adverbio (por ejemplo, las restricciones en el uso de los adverbios en -mente); (d) la función de algunos afijos y de la aposición; (e) el proceso de formación de antónimos; (f) las características de construcciones como el ablativo absoluto, los nombres contables y no contables, la comparación y la aposición; (g) la composición; (h) la derivación; (i) la concordancia lógica; (j) la conjunción; (k) la construcción; (l) elipsis; (m) el verbo; etc.

\subsection{DICCIONARIO DEL ESPAÑOL ACTUAL}

La segunda edición del Diccionario del español actual supone una revisión "exhaustiva" tanto del contenido como de la forma de las entradas del diccionario publicado inicialmente en el año 1999. Es un diccionario cuya base documental principal es literaria: 2200 libros y 400 publicaciones periódicas (p. XVI; lista completa de fuentes en apéndice III: Textos Citados, págs. 4635-4666). Además, la segunda edición ha utilizado también, con carácter complementario, "los corpus léxicos de la Real Academia Española y las ediciones de periódicos on line.” (p. XVI). El resultado es un diccionario de mayor tamaño: "el número de artículos pasa de 75000 a 83000; las acepciones, de 141000 a 198000, y las citas ascienden ahora a unas 280000.” (p. xvi)

Para el objetivo de este artículo, es relevante el análisis de los textos iniciales (pp. XVII-XXXIV), la lista A - Z (págs. 1-4602) y los apéndices finales (pp. 4607-4666). El estudio de todos ellos nos permite afirma que este diccionario también incluye todos los datos lexicográficos que ya hemos visto en los dos diccionarios anteriores. Además, ofrece algunas características lexicográficas que merecen un comentario más detallado ya que son interesantes a la hora de analizar los datos lexicográficos en una situación de producción: 
1. El diccionario ofrece registro y documentación de todas las palabras, voces y acepciones descritas en el mismo; en otras palabras, el usuario tiene acceso a "palabras de toda clase" que están suficientemente atestiguadas en la base documental del diccionario. Son palabras y acepciones que pertenecen "al uso común -activo o pasivo- del hablante.” (p. XVII) Es decir, el diccionario incluye también palabras para el uso activo de la lengua. Entre éstas, destacan la inclusión de prefijos, abreviaturas, signos y la transcripción fonológica de algunas palabras cuya pronunciación puede plantear dificultades al usuario tipo del diccionario, que, en nuestra opinión es un hablante nativo más familiarizado con el español de España que el de otros países y regiones del mundo (énfasis nuestro). En el diccionario

hay artículos que no corresponden a palabras ni a locuciones, sino a elementos prefijales. Entendemos por elemento prefijal (pref) tanto los prefijos propiamente dichos, elementos léxicos carentes de existencia independiente y que solo aparecen como miembros iniciales de palabras (por ejemplo, pre-, des-, in-), como las palabras independientes adaptadas o cambiadas formalmente para funcionar como prefijos (por ejemplo, aero-, bidro-, euro-). Hemos concedido entradas solamente a aquellos que hoy son productivos en nuestro léxico, al intervenir, combinándose con una palabra viva del idioma, en la creación espontánea de nuevas palabras, las cuales son útiles en esa ocasión y de comprensión fácil conociendo el valor de los componentes, pero que no consta que hayan acreditado aún -y quizá nunca lo hagan - su estabilidad dentro de la lengua. (Diccionario del español actual: xix)

2. El diccionario incluye un glosario de los términos lingüísticos que aparecen en los artículos, normalmente como abreviaturas en las entradas del diccionario. Este texto es importante para la producción ya que explica las funciones principales de cada término, incluyendo su comportamiento sintáctico. Por ejemplo, la abreviatura adj corresponde a adjetivo, cuya descripción gramatical en el glosario registra las tres funciones principales del mismo. Una de ellas, que es muy importante en español, es que puede fácilmente sustantivarse; esto se recoge en el diccionario con la marca "Frec n." que indica que el adjetivo aparece normalmente sustantivado y con la marca " $t b a d j "$ para indicar que el sustantivo también se usa como adjetivo.

3. El diccionario utiliza criterios diversos para enfrentarse al problema de la homonimia y la polisemia. Por ejemplo, se utiliza un criterio semántico para 
tratar como homónimo el adjetivo gentil; sin embargo, no utiliza ningún criterio para diferenciar entre cerdo como nombre o como adjetivo; finalmente utiliza un criterio gramatical para diferenciar entre poder como nombre o como verbo.

4. El diccionario también incluye en el apéndice II un modelo de conjugación de verbos divididos en (a) verbos regulares y (b) verbos irregulares. El conocimiento de las formas verbales del español es, sin duda, un ejercicio más o menos complicado y es por ello que este tipo de datos son muy necesarios para una situación de producción. Por ejemplo, en el apéndice se afirma que hay seis categorías de "verbos regulares terminados en -iar, -uar, que llevan acentuada o no acentuada la /i/ y la / u/ [1a, 1b, 1c, 1d,], así como los verbos con grupo /ai/ o / au/ en la base, que llevan articulado ese grupo en unos casos como diptongo y en otros como hiato [1e, 1f].” (p. 4631) Las categorías identificadas con símbolos como 1a, 1b, etc. aparecen también en las entradas del diccionario (ejemplo, 5 con el verbo conciliar) y ofrecen de esta forma datos necesarios para la producción:

conciliar $^{1}$ (conjug 1a) $\operatorname{tr} 1$ Poner en armonía o de acuerdo [a dos o más pers. o cosas $(c d)$ con otra(s)]. I| Gironella Millón 76: Procure usted conciliar los criterios, conseguir la unidad. b) hacer compatibles [dos o más cosas, o una(s) con otra(s)]. Torrente Djuan 33: ¿Piensas seriamente que el dogma puede ser conciliado con el evolucionismo?

$2 \approx$ el sueño $\rightarrow$ SUEÑO.

Ejemplo 5. Entrada del verbo conciliar en el Diccionario del español actual

\subsection{ZONA PREMIUM dE DiCCIONARIOS.COM}

A la zona "Premium" de Diccionarios.com (http://www.diccionarios.com/) se accede mediante suscripción. Hay varias opciones, siendo relevantes para este artículo los diccionarios de español incluidos en la pestaña "Español” e identificados como "Lengua española", "Sinónimos y antónimos", "ideológico"; también son importantes las herramientas o funcionalidades identificadas como "Editor de textos", "corrector ortográfico", “Audio” y “Conjugador verbal”.

Según la información aparecida en la pestaña "Quiénes somos", Diccionarios.com pertenece a la empresa Larousse Editorial, "que publica diccionarios, enciclopedias y 
otras obras de referencia con las marcas Larousse y Vox" (página web). No hay ninguna información sobre las características lexicográficas de los diccionarios, por lo que el análisis que presentamos a continuación se basa en el estudio que hemos realizado de algunas de sus entradas y funcionalidades. En concreto, hemos analizado la entrada conciliar seleccionando las pestañas Ideológico (Figura 1) y "Lengua española" (Figura 2):

Conciliar
VER: comité , concordia , pacificación
@ (c) Vox, marca registrada por Larousse Editorial
"conciliar" no aparece en nuestros foros

Figura 1. La entrada conciliar en Diccionarios.com (Zona Premium) (Ideológico)

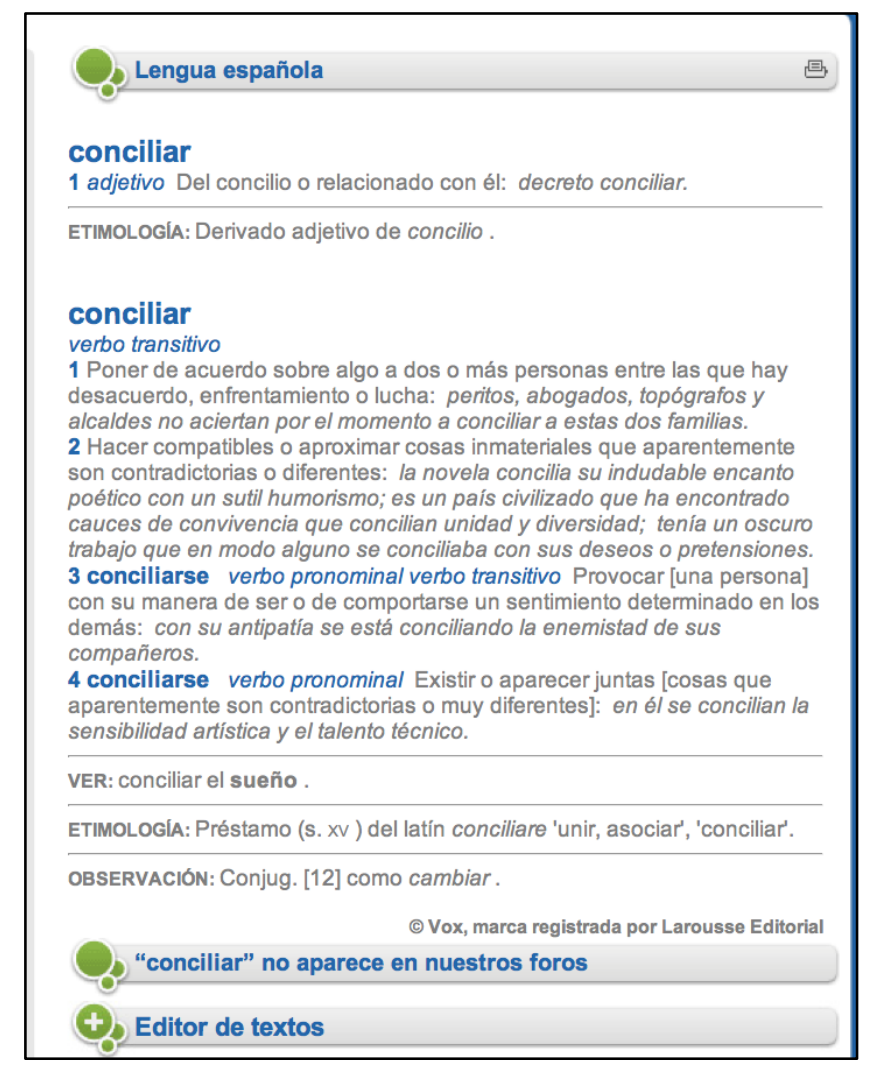

Figura 2. La entrada conciliar en Diccionarios.com (Zona Premium) (Lengua española) 
El análisis de estas figuras nos indica que Diccionarios.com (Zona Premium) incluye menos datos que los diccionarios en papel antes analizados, que el tratamiento de los datos incluidos es parecido y que no se hacen uso de tecnologías de Internet para facilitar el proceso de consulta. Por ejemplo, la descripción gramatical 3 y 4 del verbo conciliar obliga al usuario a buscar por su cuenta las implicaciones que pudiera tener en una situación de producción las marcas "verbo pronominal verbo transitivo" y "verbo pronominal". En este mismo sentido podemos situar la presencia de las valencias [una persona] y [cosas que aparentemente son contradictorias o muy diferentes] en las definiciones 3 y 4 de conciliar. En otras palabras, parece que los creadores de Diccionarios.com (zona Premium) están más interesados en lo accesorio (por ejemplo, los foros), que en lo fundamental, que es ofrecer explicaciones claras y sencillas que ayuden al usuario a utilizar los datos de la forma más rápida y fácil posible.

Como conclusión del análisis llevado a cabo en las secciones 2 y 3 puede afirmarse que hay espacio para construir un diccionario de español para la producción de textos. En nuestra opinión dicho espacio aconseja diseñar un diccionario para ser usado en una situación única (situación monofuncional) y basado en estas ideas generales:

- El diccionario debe diseñarse y construirse partiendo del significante en vez del concepto ya que la concepción de diccionarios como el Diccionario ideológico de Casares no aporta ninguna ventaja en un diccionario de Internet, que puede usar sistemas de búsquedas avanzadas (por ejemplo, búsquedas booleanas) para buscar por conceptos.

- El diccionario debe ofrecer datos lexicográficos de calidad. Entendemos por datos de calidad aquellos que describen cada uno de los significados y usos de una manera precisa y fácil de entender y de usar. Por ejemplo, nunca debe definirse trabajador como "persona que trabaja", etc. Tampoco debe dejarse al juicio del usuario decidir por su cuenta la implicación de los datos, especialmente como en el caso del ejemplo (4) por qué hay dos entradas para cantarera.

- El diccionario debe lograr que los datos lexicográficos se transformen fácilmente en información. Esto se consigue reduciendo la fatiga cognitiva que 
ocurre cuando el usuario no tiene a su disposición datos lexicográficos suficientes y bien estructurados para poder decidir lo que le interesa (por ejemplo, emplear la marca verbo transitivo sin explicar qué significa de una forma sencilla y precisa).

- El diccionario debe tener muy en cuenta el proceso de acceso a los datos con el fin de lograr que la consulta de los mismos sea lo más rápida y eficiente posible. Por ejemplo, debe ilustrarse la conjugación de un verbo español en vez de remitir al usuario a un número como se hace en la Figura 2.

- El diccionario debe estar equipado con tecnologías que bloqueen el rastro de la consulta realizada. Este es especialmente importante para usuarios (por ejemplo, una empresa) que no quieren que los rastreadores de Internet descubran los temas que interesan a la empresa, algo que puede hacerse fácilmente si la consulta en Internet deja rastros.

- El diccionario debe tender a la individualización de datos, esto puede lograrse construyendo diccionarios dinámicos con datos dinámicos, es decir diccionarios cuyos datos son diferentes según la situación.

El resultado práctico de todo lo anterior es el diseño y construcción de diccionarios de Internet como el diccionario del español para la producción de textos que veremos a continuación, primero justificando la construcción de diccionarios monolingües (sección 4) y, segundo, mostrando algunas de sus características principales.

\section{DICCIONARIOS MONOFUNCIONALES Y POLIFUNCIONALES}

En la literatura metalexicográfica relacionada con la lexicografía de Internet — ver Fuertes-Olivera, 2018; Fuertes-Olivera y Bergenholtz, 2011a; Fuertes-Olivera y Tarp, 2014) - los términos diccionario monofuncional y diccionario polifuncional se usan con frecuencia e identifican a un diccionario pensado para solucionar las necesidades de un usuario en una única situación de uso (diccionario monofuncional) o en varias situaciones de uso (diccionario polifuncional). Este planteamiento se considera un paso adelante ya que reduce la fatiga cognitiva del diccionario concebido de forma tradicional cuya característica más reconocida es que el mismo obliga al usuario a 
decidir por sí mismo los datos que puedan interesarle en cada situación de consulta y elimina la sobrecarga informativa típica de los diccionarios polifuncionales en los que el artículo del diccionario ofrece muchos más datos de los necesarios en una situación de consulta dada. Sin embargo, estas ventajas pueden mejorarse aún más y por ello autores como Bergenholtz (2011) y Tarp (2011) proponen una diferenciación más precisa tanto de usuarios como de situaciones de uso. Así, además de las cuatro situaciones de uso básicas (situación cognitiva; situación interpretativa; situación operativa y situación comunicativa), se argumenta la necesidad de establecer situaciones secundarias. Por ejemplo, para la situación comunicativa se diferencia cuatro situaciones diferentes, aunque bastante relacionadas entre sí: recepción de textos; producción de textos; corrección de textos; y traducción de textos.

En el marco de estas clasificaciones más precisas y detalladas, nos hemos planteado el diseño y construcción de un portal lexicográfico con varios tipos de diccionarios, por ejemplo, con los siguientes tipos de diccionarios, todos ellos adecuados para la producción de textos:

- Diccionario de sinónimos (y antónimos): Ofrece al usuario otra palabra en vez de la palabra conocida.

- Diccionario de búsqueda por conceptos (WordFinder en inglés) gracias al cual se ofrece al usuario la posibilidad de encontrar una palabra cuando el usuario tiene una idea más o menos vaga del significado de la palabra pero no sabe o no recuerda la palabra en cuestión. Por ejemplo encontrar la palabra cacica buscando "mujer + poder".

- Diccionario de gramática: Ayuda al usuario que está escribiendo un texto y no recuerda o desconoce la descripción gramatical, por ejemplo las flexiones y el género, de la palabra que está usando o quiere usar.

- Diccionario de ortografía: Ayuda al usuario que está escribiendo un texto y no recuerda o no sabe cómo se escribe una palabra concreta, o cómo es la separación silábica, etc.

- Diccionario de colocaciones: Ayuda al usuario cuando está escribiendo un texto y no sabe o no está seguro de la combinación o combinaciones posibles 
de la palabra que está escribiendo.

- Diccionario de formación de palabras: Ayuda al usuario cuando está escribiendo un texto y quiere información sobre raíces, sufijos, afijos, prefijos, compuestos, etc.

A todos los diccionarios que acabamos de mencionar en el listado anterior los denominamos diccionarios monofuncionales especificos reservando la denominación de diccionario monofuncional general para el Diccionario del español para producción de textos que, como veremos más adelante, incluye todos y cada uno de los datos lexicográficos de los diccionarios de la lista anterior.

\section{DICCIONARIO DEL ESPAÑOL PARA LA PRODUCCIÓN DE 'T'EX'TOS}

El Diccionario del español para la producción de textos es un integrante del portal lexicográfico Diccionarios Valladolid-UVa que esperamos poner a disposición del público en el año 2018 o 2019. En el momento de escribir este artículo, el diccionario incluye descripciones de unas 80000 acepciones. Esta cifra aumenta a una media de 30000 acepciones por año, aproximadamente.

Su concepción y desarrollo responde a cuatro ideas fundamentales. La primera de las ideas es que, como hemos visto en las secciones 2 y 3 anteriores, los diccionarios de producción son importantes desde el punto de vista lexicográfico y comercial. Creemos que ni los diccionarios ideológicos ni los diccionarios polifuncionales existentes están exclusivamente diseñados para ayudar al usuario a componer un texto, especialmente en situaciones en las que el usuario tiene dudas sobre aspectos centrales de una palabra o expresión concreta, como pueden ser los siguientes: (a) su verdadero significado; (b) usos específicos en contextos y cotextos concretos; (c) ortografía; (d) pronunciación (especialmente la pronunciación de algunas palabras con grafías y formaciones alejadas del español); (e) información gramatical, tanto la relativa a su clase gramatical y flexión como a sus propiedades combinatorias; (f) marcas diatópicas, diafásicas y diastráticas, especialmente cuando puede haber diferencias por razones geográficas, estilísticas, sociales, etc. Por esta razón, el diccionario del español para la producción de textos incluye hasta un total de 21 datos lexicográficos, todos los cuales 
pueden ser necesario en esta situación comunicativa:

1. Lema

2. Marcador de estilo del lema

3. Marcador de homonimia

4. Marcador de polisemia

5. Definición

6. Marca léxica

7. Marca de uso

8. Clase gramatical

9. Flexión recomendada (si hay varias opciones) y explicación de por qué no se recomienda otra u otras flexiones

10. Valencias (si existen)

11. Marcas y explicaciones gramaticales (si existen)

12. Marcas y explicaciones ortográficas (si existen)

13. Colocaciones (el significado de la palabra en varios contextos)

14. Ejemplos

15. Palabras relacionadas

16. Sinónimos

17. Antónimos

18. Marca de estilo de sinónimos y antónimos

19. Marca sinonímica, por ejemplo para indicar que un sinónimo determinado solo se usa en un país de Hispanoamérica

20. Marca geográfica, por ejemplo, un americanismo de México.

21. Hipervínculo, por ejemplo a un conjugador verbal de uso libre como el de WordReference.com

Las Figuras 3, 4 y 5 muestran tres entradas del diccionario correspondientes a un nombre, un verbo y una expresión. Todas ellas tienen la información gramatical básica (por ejemplo, la categoría gramatical y las flexiones), y contextual (por ejemplo, la valencia de saber de memoria), una definición precisa para cada significado (por ejemplo, las definiciones de gachupina (mujer) y de gachupin (personaje literario), sinónimos, 
antónimos, ejemplos de uso, cotextos y contextos (denominados colocaciones en este diccionario), hipervínculos (conjugación del verbo completa haciendo click en el enlace conjugación del verbo). Además, el diccionario utiliza solamente un metalenguaje claro (por ejemplo, flexiones) sin empleo de abreviaciones, códigos, símbolos, etc.

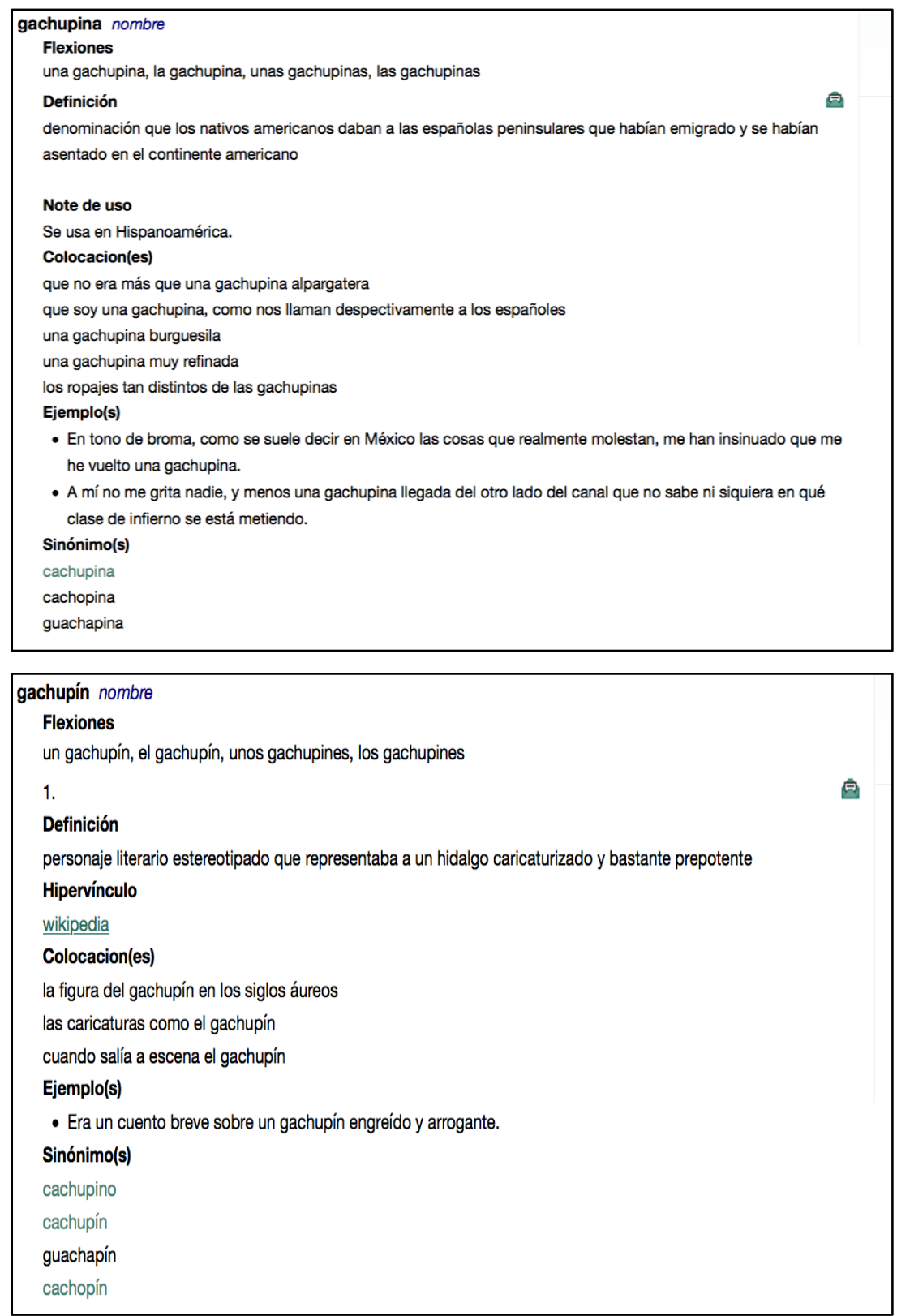

Figura 3. Entrada de gacbupin (extracto; significado 1) y gacbupina (nombre) en el Diccionario del español para la producción de textos 


\begin{tabular}{l}
\hline zarpar verbo \\
Flexiones \\
zarpo, zarpé, zarpaba, zarparé, zarparía, zarpe, zarpado, zarpando \\
Referencia gramatical \\
$\rightarrow \underline{\text { conjugación del verbo }}$ \\
Definición \\
marcharse una embarcación del lugar donde estaba fondeado, tras desanclar su ancla \\
Hipervínculo \\
conjugación del verbo \\
Colocacion(es) \\
que Salvamento sigue buscando una patera que zarpó el domingo desde Mauritania \\
que la flota zarpó rumbo al mar Rojo \\
que las nuevas embarcaciones, Yunco y Huairavo, ya zarparon desde el puerto de Nansha en China y pasarán \\
cerca 60 días en alta mar \\
que el barco zarpó a pesar de las prohibiciones por mal tiempo \\
que en 1745 zarparon para América 24 navios sueltos \\
Ejemplo(s) \\
- Una flota compuesta por 73 barcos zarpó ayer en una nueva edición de la tradicional regata Buenos Aires-Punta \\
del Este-Buenos Aires.
\end{tabular}

Figura 4. Entrada de zarpar (verbo) en el Diccionario del español para la producción de textos

saber de memoria expresión
Flexiones
sé de memoria, supe de memoria, sabía de memoria, sabré de memoria, sabría de memoria, sepa de memoria,
sabiendo de memoriasé de memoria, supe de memoria, sabía de memoria, sabré de memoria, sabría de memoria,
sepa de memoria, sabido de memoria, sabiendo de memoria
Definición
ser capaz de recordar y reproducir información de forma automática, sin necesidad de analizarla o procesarla;
puede ser información lingüística, movimientos, números, recorridos u otros
Colocacion(es)
leer una y otra vez hasta que te la sepas de memoria
que el niño sabe de memoria muchos diálogos de películas
que se sabe de memoria muchas oraciones católicas
saber de memoria la Declaración Universal de Derechos Humanos
él, que sabía de memoria su número de teléfono
Ejemplo(s)
- El preso sabe de memoria el tiempo que lleva entre rejas.
Gramática Valencias:
alguien sabe de memoria algo

Figura 5. saber de memoria (expresión) en el Diccionario del español para la producción de textos

La segunda de las ideas es que el diccionario está basado en la teoría funcional de la lexicografía (Fuertes-Olivera y Tarp, 2014; Tarp, 2008) y por ello se enfrenta a su conceptualización pensando que un diccionario es una herramienta de consulta que debe construirse para ayudar al usuario utilizando todos los medios necesarios para evitar la fatiga cognitiva que se produce cuando el usuario tiene que descifrar símbolos, 
acudir a otra entrada del diccionario para descubrir el significado, conocer la terminología lingüística compleja, etc. Por esta razón, el diccionario siempre emplea un metalenguaje lexicográfico completo (por ejemplo "nombre" en vez de "n", y lematiza como entradas independientes cachupin y cachupina ya que cada una de ellas describen realidades diferentes: en la entrada para gachupin hay un significado para "hombre" (significado específico) y un significado diferente para "persona" (significado genérico). Además, esta decisión ha tenido consecuencias lexicográficas inesperadas. Por ejemplo, para la entrada cachupina (solo hay un significado en los diccionarios españoles para cachupin, ina; ver ejemplo 1) hemos encontrados ocho significados diferentes: (a) mujer peninsular española en Hispanoamérica; (b) baile tradicional en Chile; (c) un arbusto perenne de la familia de las Malváceas; (d) flor vistosa que procede de Asica central; (e) forma coloquial de llamar a la vagina de una mujer; (f) forma coloquial de dirigirse a una perra; (g) camisa de fuerza; y (h) cartuchera. La Figura 6 muestra uno de los significados de cachupina en este diccionario. No aparece la información gramatical y de uso porque la misma siempre aparece antes de cada significado si es la misma para todos ellos o en entrada diferente si es información distinta (es decir, el diccionario utiliza la gramática de cada lema para establecer las relaciones de homonimia que pudieran existir):

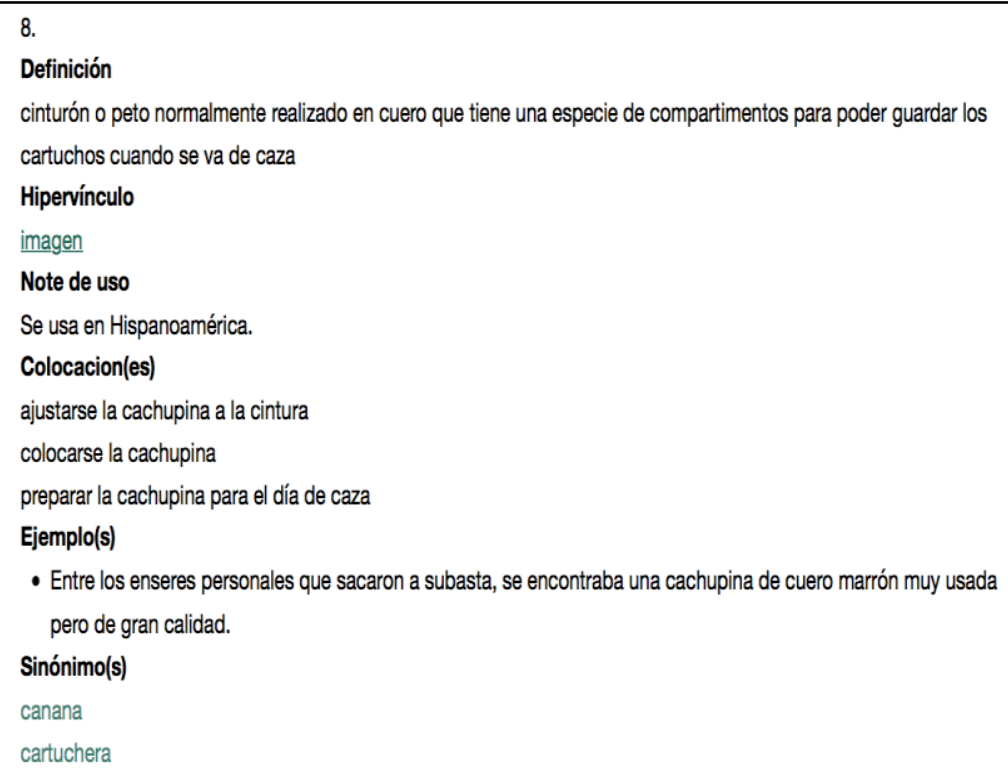

Figura 6. Uno de los significados de cachupina en el Diccionario del español para la producción de textos 
La tercera idea es que un diccionario de Internet es siempre una herramienta de información compuesta por tres componentes principales, a saber: una base de datos lexicográfica o editor utilizada para almacenar el mayor número de datos lexicográficos posibles; una interfaz, a la que acceden los usuarios (la mayoría de las personas identifican la interfaz con el diccionario de Internet); y uno o varios motores de búsqueda que se programan de forma específica para que cada uno de ellos ofrezca solamente alguno de los datos contenidos en el editor. Con esta fórmula se evita la sobrecarga informativa y se da un paso para lograr la individualización de los datos, que puede ser uno de los objetivos básicos de la lexicografía de Internet. Es decir, cada diccionario de los Diccionarios Valladolid UVa se identifica con un motor de búsqueda que ha sido programado para que el usuario reciba solamente los datos del editor que los lexicógrafos han seleccionados como adecuados para la situación cubierta por el motor de búsqueda. En el editor (también conocido como Dictionary Writing System, Abel, 2012) trabajan los lexicógrafos rellenando hasta 42 datos diferentes necesarios para describir el español general de los Diccionarios Valladolid-UVa. Durante la etapa de concepción del diccionario, los lexicógrafos junto con informáticos e ingenieros del conocimiento deciden cuáles de esos 42 datos son relevantes para una situación de producción y actúan en consecuencia. Esto significa emplear lenguajes de programación gracias a los cuales la activación del motor de búsqueda del diccionario solo recupera los datos previamente elegidos y no todos. La selección de los datos puede cambiarse fácilmente, algo que podrá hacerse una vez que el diccionario esté publicado y los lexicógrafos puedan analizar los log files y las consultas de los usuarios. Por ejemplo, puede añadirse una explicación más detallada de la gramática de algunas palabras si se juzga necesario.

Finalmente, el diccionario emplea tecnologías modernas necesarias para llevar a cabo cuatro operaciones muy importantes: (a) utiliza tecnologías para bloquear los rastros que dejan los usuarios; (b) se actualizan las entradas al minuto; (c) emplea Internet como corpus y selecciona los diversos significados de cada lema estudiando los "minitextos" de Google (Tarp y Fuertes-Olivera, 2016); (d) cada significado de cada entrada puede verse en un teléfono móvil normal con comodidad (ver la Figura 6 para uno de los significados de cachupina). 


\section{CONCLUSIONES}

El artículo se sitúa en el marco teórico de la Teoría Funcional de la Lexicografía y hace una apuesta por el diseño y construcción de herramientas de consulta monofuncionales con fines comerciales. Por ello se considera necesario el trabajo en equipo de lexicógrafos, especialistas en la temática del diccionario (por ejemplo, lingüistas para diccionarios de lengua, matemáticos para un diccionario especializado de matemáticas, etc.), informáticos e ingenieros del conocimiento. En el proyecto Diccionarios V alladolid-UV $a$, los ingenieros del conocimiento asignados al proyecto por la empresa Ordbogen A/S diseñaron, entre otras cosas, rastreadores (crawlers en inglés) que, en dos meses, lograron "encontrar" las 20000 palabras españolas más buscadas en herramientas de consulta de acceso libre en Internet. Estas 20000 palabras son el lemario básico del proyecto, que va ampliándose con datos procedentes de (a) Ngram Viewer (principalmente combinaciones de "nombre" + "adjetivo", (b) listados específicos (por ejemplo, los ríos del mundo o las serpientes venenosas), (c) proceso de construcción lexicográfica (por ejemplo, sinónimos y antónimos), y (d) corpus de acceso libre en Internet.

Dicho diccionario está pensado para ser usado en una situación de producción en la que el usuario necesita una serie de datos concretos, especialmente datos relacionados con el uso de cada una de las palabras en diferentes contextos semánticos. Además de precisar el uso y cada uno de los significados de cada lema, el diccionario lleva incorporado tecnologías de Internet que facilitan las búsquedas, eliminan la sobrecarga informativa y disminuyen al máximo posible la fatiga cognitiva típica del diccionario polifuncional en el que los datos lexicográficos necesarios deben ser seleccionados por el usuario, normalmente sin ayuda ninguna.

\section{Agradecimientos}

Este artículo se lleva a cabo en el marco del Proyecto financiado LaTeoría Funcional de la Lexicografía: Diseño y Construcción de Diccionarios de Internet (FFI2014-52462-P). Agradecemos al Ministerio de Economía y Competitividad los fondos recibidos que hacen posible esta investigación. También agradecemos a los integrantes del Seminario de Lexicografía Hispánica de la Universidad de Jaén la invitación para participar en las II Jornadas sobre investigaciones lexicográficas y lexicológicas (inLÉXICO2018) en las que presentamos algunas de las ideas contenidas en este artículo. 
SECCIÓN: LEXICOGRAFÍA

DICCIONARIOS DEL ESPAÑOL PARA LA PRODUCCIÓN DE TEXTOS

Pedro A. Fuertes-Olivera

Hening Bergenholtz

\section{BIBLIOGRAFÍA}

Abel, A. (2012). «Dictionary Writing Systems and Beyond», en Sylviane Granger y Magali Paquot (Eds.). (2012), Electronic Lexicography, Oxford: Oxford University Press, pp. 83-106.

Ahumada, I. (Dir./Ed.) (2014). Diccionario bibliográfico de la metalexicografía del español, 2006-2010, Jaén: Universidad de Jaén.

Bergenholtz, H. (2011). «Access to and Presentation of Needs-adapted Data in Monofunctional Internet Dictionaries». En Pedro A. Fuertes-Olivera y Henning Bergenholtz (Eds.), (2011a), pp. 30-53.

Bosque, I. y Barrios Rodríguez, M. ${ }^{a}$ A. (2018). «Spanish Lexicography in the Internet Era», en Pedro A. Fuertes-Olivera (Ed.), The Routledge Handbook of Lexicography. Londres y Nueva York: Routledge, pp. 636-660.

Casares, J. (1942). Diccionario ideológico de la lengua española. Madrid: Gredos.

Christensen, C. M. (1997). The Innovator's Dilemma: when New Technologies Cause Great Firms to Fail. Boston, Harvard: Business School Press.

Diccionarios.com https://www.diccionarios.com/ (Consulta: 25 de abril de 2018).

Diccionario de la lengua española (2014). Madrid, Real Academia Española. http://www.rae.es/ (Consulta: 2 de septiembre de 2017.

Fuertes-Olivera, P. A. (2016). «A Cambrian Explosion in Lexicography: Some Reflections for Designing and Constructing Specialised Online Dictionaries», en International Journal of Lexicography, 29 (2), pp. 226247.

Fuertes-Olivera, P. A. (Ed.) (2018). The Routledge Handbook of Lexicography. Londres y Nueva York: Routledge.

Fuertes-Olivera, P. A. y Bergenholtz, H. (Eds.) (2011a). E-Lexicography. The Internet, Digital Initiatives and Lexicography. Londres y Nueva York: Continuum.

Fuertes-Olivera, P. A. y Bergenholtz, H. (2011b). «Introduction. The Construction of Internet Dictionaries», en Pedro A. Fuertes-Olivera y Henning Bergenholtz (Eds.), E-lexicography. The Internet, Digital Initiatives and Lexicography. Londres y Nueva York: Continuum, pp. 1-16.

Fuertes-Olivera, P. A. y Tarp, S. (2014). Theory and Practice of Specialised Online Dictionaries. Lexicography versus Terminography. Berlin y Boston: de Gruyter.

Granger, S. y Paquot, M. (Eds.) (2012). Electronic Lexicography. Oxford: Oxford University Press.

Hackson, H. (Ed.) (2013). The Bloomsbury Companion to Lexicography. Londres y Nueva York: Bloomsbury.

Haensch, G. y Omeñaca, C. (2004). Los diccionarios del español en el siglo XXI (segunda edición). Salamanca: Universidad de Salamanca. 
SECCIÓN: LEXICOGRAFÍA

DICCIONARIOS DEL ESPAÑOL PARA LA PRODUCCIÓN DE TEXTOS

Pedro A. Fuertes-Olivera

Hening Bergenholtz

Hornby, A. S. (1963). «Introduction», en Advanced Learner's Dictionary of Current English. Oxford: Oxford University Press.

Kiesler, R. A. (2013). «Iberoromance, Syncronic lexicography», en R. H. Gouws, U. Heid, W. Schweickard, y H. E. Wiegand (Eds.), Dictionaries. An International Encyclopedia of Lexicography. Supplementary Volume: Recent Developments with Focus on Electronic and Computational Lexicography. Berlin y Boston: de Gruyter. pp. 645-653.

Marello, C. (2003). «The Bilingual Dictionary», en R. R. K. Hartmann (Ed.), Lexicography. Critical concepts II. Londres y Nueva York: Routledge, pp. 325-342.

Moliner, M. . en colaboración con J. Dacosta Esteban, A. M. ${ }^{a}$ Sánchez Mora, A. M. Marín Sánchez, y P. Villamor García (2007). Diccionario de uso del español. Tercera edición. Madrid: Gredos.

Sánchez, A. en colaboración con A. Anula, P. Cantos, L. Cerezo, J. C. Conde, M. Dueñas, F. Huerta, F. Iborra y F. Villar (2001). Gran diccionario de uso del español actual. Madrid: Sociedad General Española de Librería.

Seco, M. (2007): «Prólogo», en M. ${ }^{a}$ Moliner Diccionario de uso del español. Madrid: Gredos.

Seco, M., Andrés, O. y Ramos, G. en colaboración con M. ${ }^{a}$ T. de Unamuno, J. A. Villafáñez y C. Domínguez (2011). Diccionario del español actual. Segunda edición. Madrid: Aguilar.

Summers, D. (Ed.) (1993). Longman language activator. For upper intermediate - advanced learners. Harlow: Longman.

Tarp, S. (2008): Lexicography in the Borderland between Knowledge and Non-Knowledge: General Lexicographical Theory with Particular Focus on Learners' Lexicography. Tübingen: Niemeyer.

Tarp, S. (2011). Lexicographical and Other e-Tools for Consultation Purposes: Towards the Individualization of Needs Satisfaction", en Pedro A. Fuertes-Olivera y Henning Bergenholtz (Eds.), (2011a), pp. 5570.

Tarp, S. y Fuertes-Olivera, P. A. (2016). «Advantages and Disadvantages in the Use of Internet as a Corpus: The Case of the Online Dictionaries of Spanish Valladolid-UVa», en Lexikos 26, pp. 273-295.

WordReference. http:/ / www.wordreference.com/es/ (Consulta: 25 de abril de 2018). 